\title{
PI3K Inhibitors Reverse the Suppressive Actions of Insulin on CYP2E1 Expression by Activating Stress-Response Pathways in Primary Rat Hepatocytes
}

\author{
JASPREET S. SIDHU, FEI LIU, SEAN M. BOYLE, and CURTIS J. OMIECINSKI \\ Department of Environmental Health, University of Washington, Seattle, Washington \\ Received November 14, 2000; accepted January 31, 2001 \\ This paper is available online at http://molpharm.aspetjournals.org
}

\begin{abstract}
Insulin-associated signaling pathways are critical in the regulation of hepatic physiology. Recent inhibitor-based studies have implicated a mechanistic role for phosphatidylinositol 3' kinase (PI3K) in the insulin-mediated suppression of CYP2E1 mRNA levels in hepatocytes. We investigated the dose dependence for this response and for the effects of insulin and extracellular matrix on PI3K signaling and CYP2E1 mRNA expression levels using a highly defined rat primary hepatocyte culture system. The PI3K inhibitors wortmannin and LY294002 stimulated stress-activated protein kinase/c-Jun $\mathrm{NH}_{2}$-terminal kinase (SAPK/JNK) and p38 mitogen-activated protein kinase (MAPK) phosphorylation in a rapid and concentration-dependent manner that paralleled the inhibition of protein kinase $B$ (PKB) phosphorylation. Although PI3K inhibitors reversed the sup-
\end{abstract}

pressive effects of insulin on CYP2E1 expression, these effects only occurred at concentrations well in excess of those required to achieve complete inhibition of PKB phosphorylation. These same concentrations produced cytotoxic responses as evidenced by perturbed cellular morphology and elevated release of lactate dehydrogenase. Wortmannin-mediated activation of the SAPK/JNK and p38 MAPK pathways also resulted in the mobilization of activator protein-1 complex to the nuclear compartment. We conclude that the suppression of CYP2E1 mRNA expression by insulin is not directly associated with PI3K-dependent pathway activation, but rather is linked to a cytotoxic response stemming from acute challenge with $\mathrm{PI} 3 \mathrm{~K}$ inhibitors.
Insulin is known to regulate a number of diverse metabolic functions, including amino acid transport, glucose homeostasis, and cell growth and differentiation (O'Brien and Granner, 1996). This myriad of physiological effects is largely mediated through the activation of the trans-membrane insulin receptor tyrosine kinase, leading to the stimulation of several intrinsic tyrosine kinase domains and the resultant phosphorylation of the insulin receptor substrate family of proteins (Avruch, 1998). The downstream cascade is further completed via the recruitment and activation of other intracellular signal-transducing proteins [e.g., members of the Ras-MAPK (Chuang et al., 1994) and phosphatidylinositol 3'-kinase (PI3K) pathways (Hara et al., 1994)].

The resultant PI3K-mediated phosphorylation of phosphoinositides on the D-3 position leads to the generation of secondary signaling products (Klippel et al., 1997) that act on multiple downstream effectors and target proteins. The latter includes various serine/threonine and tyrosine kinases as

This study was supported by the National Institute of General Medical Sciences (GM32281). C.J.O. is a Burroughs Wellcome Fund Toxicology Scholar. well as cytoskeletal components. Recent evidence is highly supportive of the role of the serine/threonine protein kinase B (PKB, the cellular analog of the retroviral proto-oncogene $\mathrm{v}$-Akt) as the direct downstream effecter of PI3K action (Bellacosa et al., 1991). Similar to PI3K, the activity and resultant phosphorylation of PKB is regulated by growth factors such as insulin and insulin-like growth factor-1 (Alessi et al., 1996). PKB in turn plays pivotal roles in the control of cell differentiation, proliferation, and survival (Kandel and Hay, 1999). Furthermore, PKB has recently been shown to phosphorylate various downstream target proteins that stimulate survival signals in cells and hence protect against apoptosis (Franke et al., 1997).

Therefore, any disruption of insulin-associated signaling pathways, such as that observed in diabetes, fasting, or obesity (O'Brien and Granner, 1996; Pessin and Saltiel, 2000), typically results in drastically altered metabolic function with associated long-term clinical manifestations. In addition to the classical pathophysiological changes associated with abnormal glucose homeostasis, diabetes also results in elevation of the expression of various genes, primarily in the

ABBREVIATIONS: MAPK, mitogen-activated protein kinase; PI3K, phosphatidylinositol 3'-kinase; PKB, protein kinase B; ECM, extracellular matrix; SAPK, stress-activated protein kinase; JNK, c-Jun $\mathrm{NH}_{2}$-terminal kinase; BSA, bovine serum albumin; Dex, dexamethasone; LDH, lactate dehydrogenase; EMSA, electrophoretic mobility shift assay; RT, room temperature; AP-1, activator protein-1. 
liver, involved in the metabolism of endogenous and exogenous substances (Zaluzny et al., 1990). For example, significant alterations are observed with members of the cytochrome P450 gene family (Nelson et al., 1996), in particular for CYP2E1 (Lieber, 1997), a gene that is induced prototypically by various chemicals such as ethanol, acetone, and ketone bodies (Kraner et al., 1993). Investigators have causatively linked changes in metabolic function seen in diabetic liver, such as increased levels of ketone bodies, to accumulation of CYP2E1 mRNA levels (Dong et al., 1988).

The liver is a primary target organ of diabetes, and recent improvements to primary hepatocyte culture-based models have facilitated the characterization of mechanisms of insulin action and the resulting physiology of the diabetic disease state. Using such a model system, it was reported that insulin suppresses the expression of CYP2E1 (Woodcroft and Novak, 1999a) through specific mechanisms (Woodcroft and Novak, 1999b) implicating PI3K and src kinase pathways.

In the present study, we used a highly defined rat primary hepatocyte culture system (Sidhu et al., 1993; Sidhu and Omiecinski, 1999) to investigate the effects of insulin and extracellular matrix (ECM) on the regulation of hepatocyte gene expression via PI3K signaling pathways. Extensive dose-response studies were conducted for several PI3K inhibitors, wortmannin (Powis et al., 1994), LY294002 (Vlahos et al., 1994), quercetin (Matter et al., 1992), and the broadspectrum protein kinase inhibitor staurosporine (Meggio et al., 1995). Wortmannin and LY294002 were shown to stimulate stress-activated protein kinase/c-Jun $\mathrm{NH}_{2}$-terminal kinase (SAPK/JNK) and p38 mitogen-activated protein kinase (p38 MAPK) phosphorylation in a concentration-dependent manner that paralleled the inhibition of PKB phosphorylation by each inhibitor. We demonstrate that insulin-mediated suppression of CYP2E1 mRNA expression is not associated with the activation of a PI3K-dependent pathway. Rather, the CYP2E1 response is probably linked to the activation of intracellular stress pathways and associated cytotoxicity stemming from acute challenge with elevated concentrations of PI3K inhibitors. The results of our studies underscore the critical role of insulin as a modulator of CYP2E1 and albumin gene expression and in the maintenance of cellular integrity.

\section{Experimental Procedures}

Cell Culture Materials and Chemicals. All cell culture media and Trizol were obtained from Life Technologies, Inc. (Grand Island, NY). Matrigel, ITS + [insulin, transferrin, selenium, bovine serum albumin (BSA), and linoleic acid], and $\mathrm{Nu}$-Serum were obtained from Becton Dickinson (Bedford, MA). Transferrin, selenium, and BSAlinoleic acid were obtained from Sigma Chemical (St. Louis, MO). Collagenase (type 1) was obtained from Worthington Biochemicals (Lakewood, NJ). Tissue culture-treated plastic dishes (100 mm; Falcon Plastics, Oxnard, CA) were obtained from Becton Dickinson (Franklin Lakes, NJ). Dexamethasone (Dex; 9 $\alpha$ fluoro-16 $\alpha$ methyl$11 \beta, 17 \alpha, 21$-trihydroxy-1,4-pregnadiene-3,20-dione), and insulin (bovine) were obtained from Sigma Chemical. Quercetin, LY294002, wortmannin, and staurosporine were obtained from Alexis Chemicals (San Diego, CA). The lactate dehydrogenase (LDH)-based cell cytotoxicity kit was obtained from Promega (Madison, WI).

Isolation and Culture of Hepatocytes. Rat hepatocytes were isolated from adult Sprague-Dawley male rats (Simenson Labs, Gilroy, CA) by a two-step collagenase perfusion in situ and cultured with modification (Sidhu and Omiecinski, 1995b, 1999) of a protocol described previously (Sidhu et al., 1993). Briefly, cells were plated on tissue culture-treated plastic dishes in Williams' E medium in the presence of $10 \% \mathrm{Nu}$-Serum and $100 \mathrm{nM}$ Dex. Cells were allowed to attach for $3 \mathrm{~h}$ before switching to serum-free medium (Williams' $\mathrm{E}$, $25 \mathrm{nM}$ Dex, $6.25 \mu \mathrm{g} / \mathrm{ml}$ transferrin, $6.25 \mathrm{ng} / \mathrm{ml}$ selenium, $1.25 \mathrm{mg} / \mathrm{ml}$ BSA, $5.35 \mu \mathrm{g} / \mathrm{ml}$ linoleic acid, and variable concentrations of insulin). Subsequently, cells were cultured in the latter formulation with daily medium changes.

Matrigel Overlay. A dilute concentration ( $233 \mu \mathrm{g} / \mathrm{ml}$, final concentration) of ECM (Matrigel) was added as an overlay (Sidhu et al., 1993), $4 \mathrm{~h}$ after plating and after the first medium change.

Chemical Treatments. Unless otherwise stated, cells were cultured for $72 \mathrm{~h}$ in the absence $(0 \mathrm{nM})$ or presence of insulin $(1,10$, or $100 \mathrm{nM}$ ). At this point, insulin was removed and washed from cells before the addition of various concentrations of PI3K inhibitors (wortmannin, LY294002, quercetin). After a 1-h treatment with inhibitor, insulin was added back at the previously stated concentrations for a further $24 \mathrm{~h}$, at which point total RNA was isolated and analyzed as detailed below. In other experiments, cells were cultured completely in the absence of insulin for the initial $48 \mathrm{~h}$. Cells were then treated with varying concentrations of PI3K inhibitors for $1 \mathrm{~h}$ before the readdition of insulin $(0,1,10,100 \mathrm{nM})$ for a further $24 \mathrm{~h}$. Total RNA was isolated and analyzed as stated below. Representative data are shown from multiple experiments performed independently with five different hepatocyte preparations.

RNA Analysis. Total RNA was isolated using Trizol as described previously (Sidhu and Omiecinski, 1999) and analyzed by slot-blot hybridization as described previously (Sidhu and Omiecinski, 1995b).

cDNA Hybridizations. A cDNA probe specific for rat CYP2E1 was derived as a polymerase chain reaction product of rat liver cDNA using the following forward and reverse primer pairs: forward primer, 5'-GGATGTGACTGACTGTCTCC-3' and reverse primer, 5' TGGGGTAGGTTGGAAGGGAC-3'. The resulting polymerase chain reaction product was radiolabeled using the DECAprime DNA labeling kit ( $>3000 \mathrm{Ci} / \mathrm{mmol}$; PerkinElmer Life Science Products, Boston, MA). Hybridization was preformed essentially as described previously (Sidhu and Omiecinski, 1995b), expect conducted at $60^{\circ} \mathrm{C}$ in the absence of formamide.

Determination of LDH Release. Levels of LDH were determined in triplicate in the extracellular medium, spectrophotometrically, as per manufacturer's instructions (Promega).

Phosphorylation Analysis of PKB, SAPK/JNK, and p38 MAPK. Primary hepatocytes were cultured for 48 to $72 \mathrm{~h}$ in the absence of insulin before exposure of varying concentrations of the PI3K inhibitors for $1 \mathrm{~h}$ followed by the addition of insulin $(0,1,10$, or $100 \mathrm{nM})$. Controlled time points were taken and cell extracts were prepared and analyzed by SDS-polyacrylamide gel electrophoresis as described previously (Sidhu and Omiecinski, 1998). The membranes were probed (manufacturer's blotting instructions) with phosphospecific antibodies, which recognize the phosphorylated state of PKB (Akt), SAPK/JNK, and p38 MAPK, respectively (New England Biolabs, Beverly, MA). Treatments were normalized in parallel by assessment of protein loading using phosphorylation-independent antibodies against Akt ( $\alpha$-Akt), SAPK/JNK, and p38 MAPK. The membranes were then incubated with a goat anti-rabbit horseradish peroxidase-conjugated secondary antibody (1:2000 dilution). The ECL enhanced chemiluminescence system (Amersham Pharmacia Biotech, Piscataway, $\mathrm{NJ}$ ) was used to visualize specific immunoreactive proteins.

Electromobility Shift Assays (EMSAs). A double-stranded consensus oligonucleotide for activated protein-1 (AP-1) (5'-cgcttgatgactcagccggaa-3'; Santa Cruz Biochemicals, Santa Cruz, CA) was end-labeled with $\left[\gamma_{-}{ }^{32} \mathrm{P}\right]$ ATP using T4 polynucleotide kinase (Amersham Pharmacia Biotech). Nucleotides in bold type represent mutated sites (ca $\rightarrow$ tg). Wild-type and mutant oligonucleotides were used in 80 -fold excess to the radiolabeled probe in competition experiments. Hepatocyte nuclear protein was isolated, after a 4-h treatment, using the NuPer kit (Pierce, Rockford, IL). Ten micro- 
grams of nuclear protein was incubated in binding buffer $(50 \mathrm{mM}$ Tris-Cl, pH 7.5, $2.5 \mathrm{mM}$ dithiothreitol, $2.5 \mathrm{mM}$ EDTA, $250 \mathrm{mM} \mathrm{NaCl}$, $5 \mathrm{mM} \mathrm{MgCl}_{2}$, and $20 \%$ glycerol) for $15 \mathrm{~min}$ at RT. Five nanograms of the labeled probe $(\sim 20,000 \mathrm{cpm})$ was added and incubated for an additional $15 \mathrm{~min}$ at RT. For super shift analysis, $2 \mu \mathrm{g}$ of antibody directed against the carboxyl terminal of $c$-fos (Santa Cruz Biochemicals) was added and incubated at RT for a further $15 \mathrm{~min}$. Nuclear protein complexes were resolved on 5\% Tris/borate/EDTA gels (BioRad, Hercules, CA), dried, and subjected to autoradiography. Protein concentration was determined with bovine serum albumin as standard using a commercial kit (bicinchinonic acid protein assay reagent; Pierce).

\section{Results}

The primary aim of the present study was to examine the potential involvement of PI3K signaling pathways in modulating the effects of insulin on hepatic gene expression, in particular, CYP2E1. Our previous studies have demonstrated the importance of culture environment in maintaining highly differentiated function of hepatocytes in vitro. Therefore, initially we examined the potential interplay of hepatocyte differentiation status with insulin responsiveness.

Effect of ECM Overlay, Insulin, and Wortmannin on CYP2E1 and Albumin mRNA Expression. Primary rat hepatocytes were cultured in the presence or absence of an ECM overlay under varying concentrations of insulin $(0,1,10$ $\mathrm{nM}$ ) for the initial $48 \mathrm{~h}$ after plating. Cells were washed extensively to remove insulin and subsequently treated with wortmannin at concentrations ranging from 0 to $250 \mathrm{nM}$ for $1 \mathrm{~h}$ before the readdition (where applicable) of insulin, and then cultured for a further $24 \mathrm{~h}$. Total RNA was isolated and examined for the expression level of CYP2E1 and albumin mRNA. The results of these experiments are shown in Fig. 1. The data demonstrate that maintenance of stable levels of CYP2E1 mRNA, equivalent to that observed in freshly isolated hepatocytes (ex vivo), was highly dependent on the presence of an ECM overlay and that CYP2E1 expression

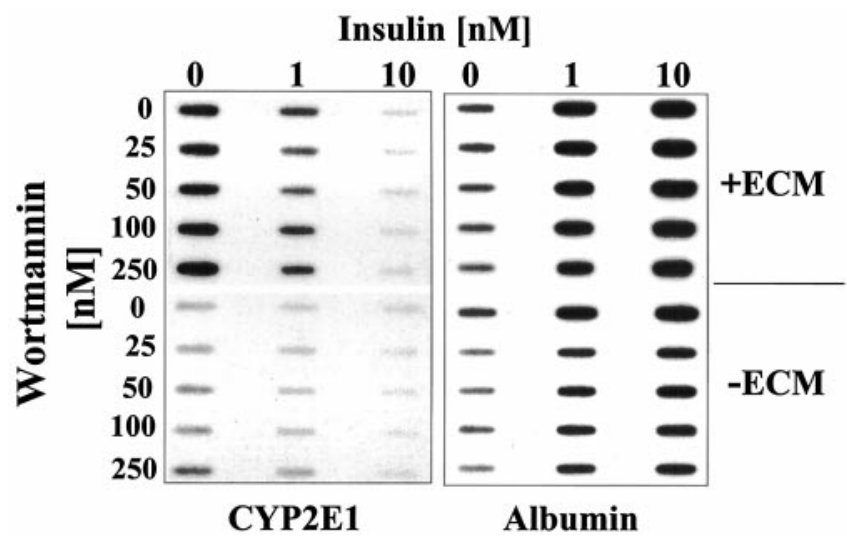

Fig. 1. Effect of ECM overlay, insulin concentration, and wortmannin treatments on CYP2E1 and albumin mRNA expression. Primary rat hepatocytes were cultured for $48 \mathrm{~h}$ under the stated insulin concentrations (nanomolar) and in the absence $(-)$ or presence $(+)$ of an ECM overlay. Subsequently, cells were washed with insulin-free medium several times and treated with a range of wortmannin concentrations (25$250 \mathrm{nM}$ ) for $1 \mathrm{~h}$ before the readdition of the previous insulin concentrations. These treatments were continued for a further $24 \mathrm{~h}$ and then total RNA was isolated and evaluated by slot-blot analysis as described under Experimental Procedures. CYP2E1 and albumin mRNA expression are shown. Ribosomal 18S RNA hybridization levels were used as normalization standards to demonstrate equal loading of RNA. was completely suppressed by the addition of $10 \mathrm{nM}$ insulin. Maintenance of hepatocytes in $1 \mathrm{nM}$ insulin resulted in $<50 \%$ of the ex vivo CYP2E1 mRNA levels. Identical results were obtained when examining CYP2E1 protein levels (data not shown). We compared these effects to that of a prototypical liver marker, the expression of albumin mRNA. The albumin gene has been shown to undergo positive regulation by both insulin (Kimball et al., 1995) and ECM addition (Bissell et al., 1990). The supportive effect of both ECM overlay and insulin concentration on albumin expression is also demonstrated in these analyses. It is noteworthy that the wortmannin exposures were completely ineffective in reversing the repressive effects of insulin on CYP2E1 mRNA expression.

Examining Wortmannin Dose-Response Requirements for Inhibiting PI3K/PKB-Dependent Phosphorylation. We examined a range of wortmannin concentrations to determine its inhibition profile on insulin-stimulated phosphorylation of PKB. Hepatocytes were deprived of insulin for $72 \mathrm{~h}$ before their incubation with increasing concentrations of wortmannin for $30 \mathrm{~min}$. Insulin was then reintroduced (1-10 $\mathrm{nM}$ ) and the cells were incubated for a subsequent $30 \mathrm{~min}$, total cell extracts prepared (see under Experimental Procedures), and examined by Western immunoblot for the phosphorylation of PKB using a phospho-specific antibody as well as normalized to total immunoreactive PKB ( $\alpha$-Akt). Wortmannin produced a dose-dependent inhibition of PKB phosphorylation, such that $>50 \%$ inhibition was observed at a wortmannin concentration of $50 \mathrm{nM}$, whereas $>95 \%$ inhibition was effected at $100 \mathrm{nM}$ (Fig. 2A). Higher concentrations of this inhibitor yielded no further response.

Reversal of Insulin-Mediated Suppression of CYP2E1 mRNA Expression at Elevated Wortmannin Concentration. Because PKB phosphorylation was inhibited within a range of wortmannin concentrations that were ineffective in reversing insulin's suppression of CYP2E1 mRNA levels, we extended the dose range to include higher concentrations used in the literature (Woodcroft and Novak, 1999b). Hepatocytes were cultured in the complete absence of insulin for $72 \mathrm{~h}$, and then treated for $1 \mathrm{~h}$ with wortmannin $(0-10 \mu \mathrm{M})$ before the addition of insulin (0 or $10 \mathrm{nM})$ for a further $24 \mathrm{~h}$. Total RNA was again isolated and examined by slot-blot hybridization for CYP2E1 mRNA levels (Fig. 2B). Again, wortmannin treatment did not reverse the suppression by insulin of CYP2E1 expression at levels of inhibitor $<1$ $\mu \mathrm{M}$. However, a complete reversal was achieved at $10 \mu \mathrm{M}$ wortmannin, a level far in excess $(\sim 100$-fold $)$ of that required to completely inhibit PKB phosphorylation. The level of CYP2E1 mRNA that resulted from the $10 \mu \mathrm{M}$ wortmannin treatments was equivalent to that observed in freshly isolated cells before plating (discussed below for other treatments). Interestingly, wortmannin alone produced a concentration-dependent stimulation of CYP2E1 mRNA expression in cells completely deprived of insulin, despite the complete lack of PKB phosphorylation in the absence of hormone (also discussed below).

Effect of LY294002 on Reversal of Insulin-Mediated Suppression of CYP2E1 mRNA Expression. We next examined the dose dependence of reversal of insulin-mediated suppression of CYP2E1 with another inhibitor of PI3K, LY294002 (Vlahos et al., 1994). As with wortmannin treatments, cells were cultured in the complete absence of insulin 
for $72 \mathrm{~h}$ before the addition of LY294002 for 30 min followed by treatment with $10 \mathrm{nM}$ insulin for a subsequent $30 \mathrm{~min}$. Total cell extracts were prepared and examined for PKB phosphorylation (Fig. 3A). LY294002 produced a dose-dependent inhibition of insulin-mediated phosphorylation of PKB, resulting in $>50 \%$ inhibition at a concentration of $10 \mu \mathrm{M}$. Relatively complete inhibition was obtained at a concentration of $25 \mu \mathrm{M}$. However, the PKB effects produced by this agent did not correlate with the corresponding lack of reversal of insulin-mediated suppression of CYP2E1 mRNA levels (Fig. 3B). In fact, LY294002 additions produced only marginal effects on CYP2E1 expression in the presence of insulin. As was the case with wortmannin, exposure of cells to high levels of LY294002 (>25 $\mu \mathrm{M})$ enhanced CYP2E1 mRNA expression even when completely deprived of insulin.

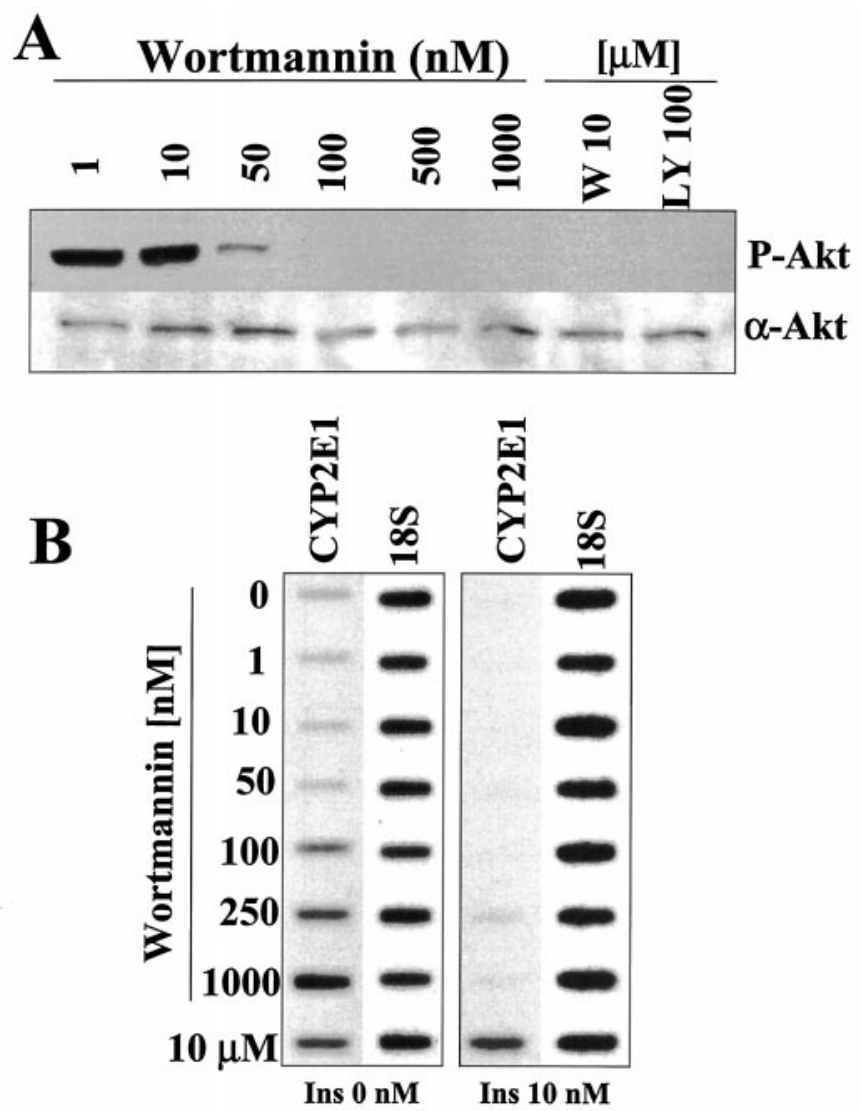

Fig. 2. Effect of wortmannin concentration on insulin-mediated phosphorylation of PKB and suppression of CYP2E1 mRNA expression in primary rat hepatocytes. Primary rat hepatocytes were cultured for $72 \mathrm{~h}$ in insulin-free medium in the presence of an ECM overlay. Subsequently, cells were pretreated with wortmannin (1-1000 nM, $10 \mu \mathrm{M})$ or LY294002 $(10 \mu \mathrm{M})$ for $30 \mathrm{~min}$ before treatment with insulin for $30 \mathrm{~min}$. Total cell extracts were prepared as stated under Experimental Procedures and analyzed by Western blot analysis (A). Phosphorylation status of PKB (P-Akt) was assessed using a phosphospecific antibody to Akt (Ser473). The level of immunoreactive protein was assessed with a phosphorylation-independent antibody to PKB $(\alpha$-Akt). Under similar conditions, the effect of wortmannin concentration was assessed on insulin-mediated suppression of CYP2E1 mRNA expression. Primary rat hepatocytes were cultured for $72 \mathrm{~h}$ in insulin-free medium in the presence of an ECM overlay. Subsequently, cells were pretreated with wortmannin (1-1000 $\mathrm{nM}, 10 \mu \mathrm{M})$ for $1 \mathrm{~h}$, followed by treatment with $(10 \mathrm{nM})$ or without insulin $(0 \mathrm{nM})$ for a further $24 \mathrm{~h}$. Total RNA was isolated and evaluated by slot-blot analysis as described under Experimental Procedures. The level of CYP2E1 mRNA expression and the corresponding ribosomal 18S RNA is shown (B).
Effect of Wortmannin Treatments on Hepatocyte Morphology. We asked whether there was any evidence for cytotoxicity associated with the use of elevated concentrations of wortmannin. With such exposures, there were consistent observations of perturbations in the morphological integrity of cultured hepatocytes. These effects are clearly evident in the representative photomicrographs (Fig. 4). Wortmannin treatments, resulted in a dose-dependent disruption of the cuboidal network and bile canalicular structures associated with highly differentiated hepatocytes. Furthermore, we observed a pronounced cytoplasmic condensation (Fig. 4, arrows in panels) at inhibitor concentrations $>1 \mu \mathrm{M}$. Increasing the concentration to $10 \mu \mathrm{M}$ resulted in an adverse rounding up of cellular structure (Fig. 4, arrows), probably associated with acute cellular cytotoxicity. Similar results were obtained for the morphological perturbations associated with elevated concentrations of LY294002 (>25 $\mu \mathrm{M}$; data not shown).

Stimulation of SAPK/JNK and p38 MAPK Phosphorylation by Wortmannin and LY294002. We next attempted to assess whether the compromised cellular morphology observed from the high concentrations of wortmannin might result from activation of hallmark signaling cascades involved in mediating chemical stress. At the $100 \mathrm{nM}$ wortmannin concentration that produced complete inhibition of $\mathrm{PKB}$ phosphorylation, we detected a converse dose-dependent stimulation of phosphorylation of SAPK/JNK and p38 MAPK (Fig. 5). A similar dose-response was also observed for LY294002 (data not shown). Results using $100 \mu \mathrm{M}$ LY294002 are shown for comparison (Fig. 5).

Subsequently, we examined a classic marker of cytotoxicity

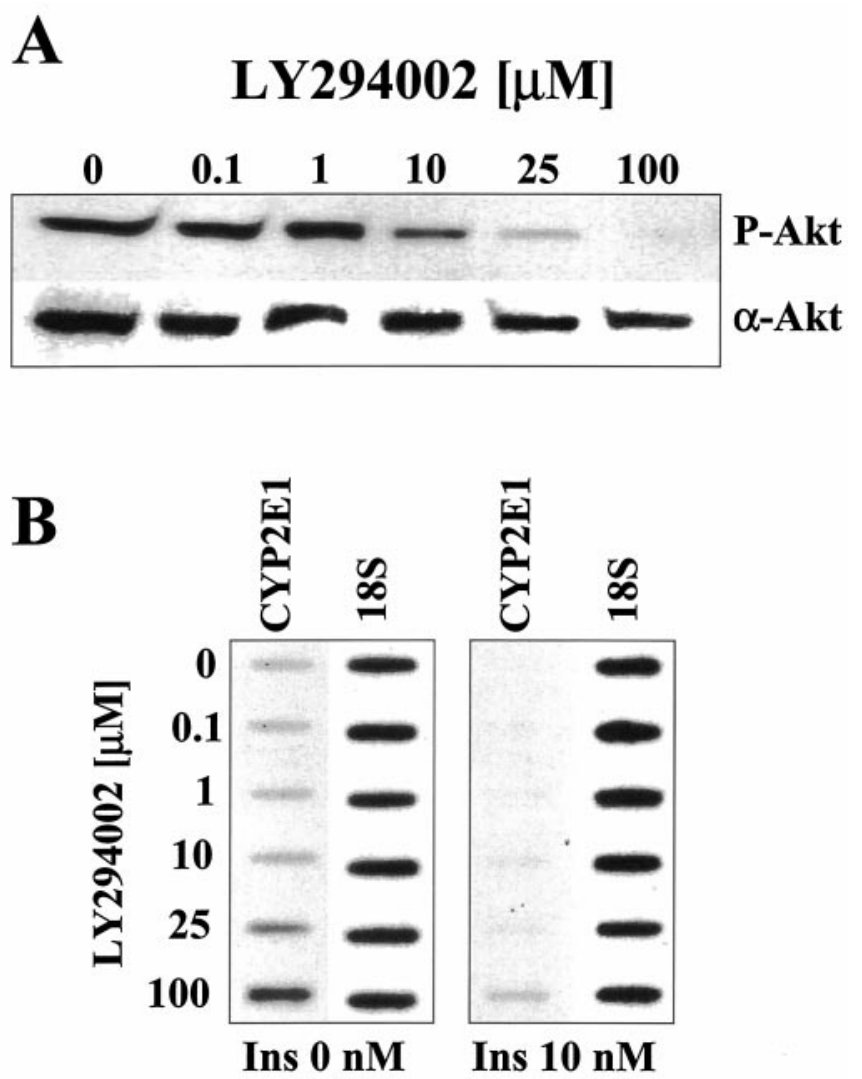

Fig. 3. Effect of LY294002 concentration on insulin-mediated phosphorylation of PKB and suppression of CYP2E1 mRNA expression in primary rat hepatocytes. For explanation of treatments, refer to Fig. 2. 
in treated cells by measurement of the release of cytosolic $\mathrm{LDH}$ into the medium. LDH release was determined spectrophotometrically in the extracellular medium $24 \mathrm{~h}$ after treatment over a range of wortmannin or LY294002 concentrations, in the presence or absence of insulin $(0,1$, or $10 \mathrm{nM})$. A dose-dependent increase in LDH leakage was detected as a function of wortmannin concentration (Fig. 6A; similarly for

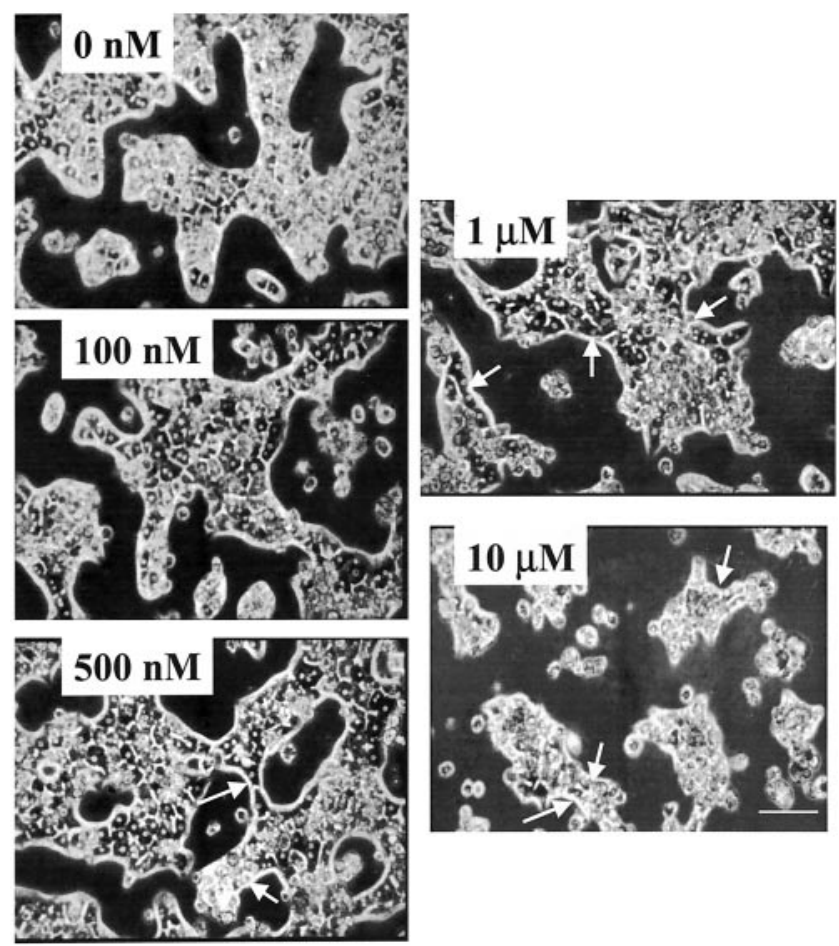

Fig. 4. Effect of wortmannin treatments on hepatocyte morphology. Primary rat hepatocytes were cultured and treated as stated in Fig. 1. The effect of wortmannin treatments for $24 \mathrm{~h}$, in the presence of insulin (10 $\mathrm{nM}$ ), on the representative morphology (magnification, $20 \times$ ) of primary rat hepatocytes is shown. Scale bar, $50 \mu \mathrm{m}$. Arrows identify evidence of perturbed morphology: condensed cytoplasm and rounded-up cells, attributed to cytotoxicity.

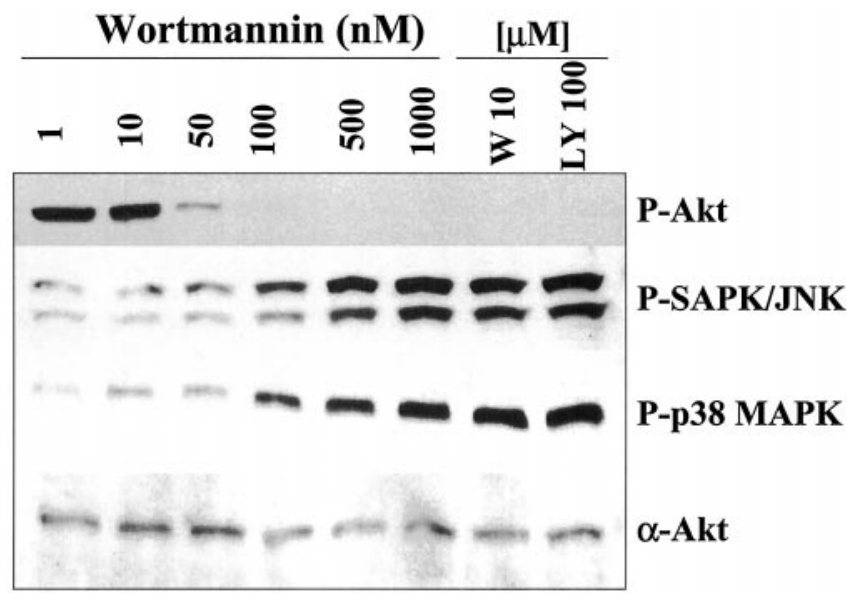

Fig. 5. Effect of wortmannin and LY294002 treatments on PKB, SAPK/ JNK, and p38 MAPK phosphorylation. Primary rat hepatocytes were cultured and pretreated with inhibitors for 30 min followed by $10 \mathrm{nM}$ insulin for an additional $30 \mathrm{~min}$. The phosphorylation status of PKB, SAPK/JNK, and p38 MAPK was assessed using phospho-specific antibodies directed against PKB (P-Akt, Ser473), SAPK/JNK (Thr183/Tyr185), and p38 MAPK (Thr180/Tyr182) as stated under Experimental Procedures. The level of immunoreactive protein was assessed with a phosphorylation-independent antibody to $\mathrm{PKB}(\alpha$-Akt).
LY294002; data not shown). The effect was most pronounced in cells deprived of insulin. Addition of insulin seemed protective in that insulin supplementation reduced the level of $\mathrm{LDH}$ released. However, hormone additions did not fully relieve the wortmannin-associated toxicity.

We then determined whether anisomycin, a more prototypical activator of the SAPK/JNK pathway, could mimic the cytotoxicity and effects of wortmannin and/or LY294002 on CYP2E1 mRNA levels. First, we examined the dose-dependent stimulation of SAPK/JNK phosphorylation with this agent. As shown in Fig. 6A, a saturating stimulation resulted at $10 \mu \mathrm{M}$ anisomycin, a concentration we demonstrated previously to potently activate the SAPK/JNK pathway and inhibit de novo protein synthesis (Sidhu and Omiecinski, 1998). The latter concentration was then tested to determine whether a similar increase in cytotoxicity and resulting reversal of CYP2E1 suppression was also associated with anisomycin. However, anisomycin treatment, relative to control, did not result in detectable $\mathrm{LDH}$ release from the cells (Fig. 6B), nor reverse insulin-mediated suppression of CYP2E1 mRNA expression (Fig. 6C). Furthermore, at this concentration, anisomycin was without any apparent adverse effect on levels of albumin mRNA (Fig. 6D) or hepatocyte morphology (data not shown).

Effect of Quercetin on Insulin-Mediated Suppression of CYP2E1 mRNA Expression. To further confirm the lack of involvement of PI3K/PKB in the insulin-mediated suppression of CYP2E1 expression, cells were treated with increasing concentrations of the natural flavonoid quercetin. This agent is the parent compound of LY294002 and has been shown to exert a potent inhibition of the PI3K-dependent pathway (Matter et al., 1992). Indeed, when cells were pre-
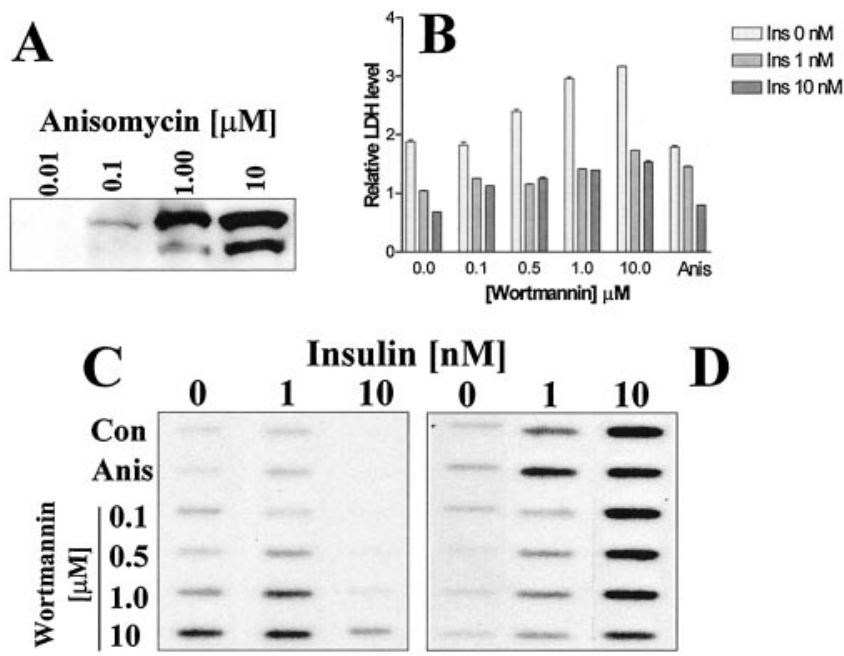

Fig. 6. Effect of wortmannin and anisomycin on levels of LDH release, SAPK/JNK phosphorylation and reversal of insulin-mediated suppression of CYP2E1 mRNA expression. Primary rat hepatocytes were cultured for $72 \mathrm{~h}$ in insulin-free medium in the presence of an ECM overlay. Subsequently, cells were treated with a range of wortmannin concentrations $(0.1-10 \mu \mathrm{M})$ or anisomycin $(10 \mu \mathrm{M})$ for $60 \mathrm{~min}$ followed by treatment with insulin $(0,1,10 \mathrm{nM})$ for a further $24 \mathrm{~h}$. The dose-dependent stimulation of SAPK/JNK phosphorylation (A) for anisomycin $(0.01-10 \mu \mathrm{M})$ treatments for $30 \mathrm{~min}$ was assessed at the same time culture time point. Release of LDH into the medium was measured $24 \mathrm{~h}$ after treatment with wortmannin and/or anisomycin as stated under Experimental Procedures and is shown in B. Total RNA was isolated and examined by slot-blot hybridization for the level of CYP2E1 (C) and albumin mRNA expression (D). Ribosomal 18S RNA hybridization levels were used as normalization standards to demonstrate equal loading of RNA (data not shown). 
treated with a range of concentrations of quercetin (Fig. 7A), we observed a complete inhibition of $\mathrm{PKB}$ phosphorylation at $25 \mu \mathrm{M}$ (also $10 \mu \mathrm{M}$; data not shown), relative to wortmannin (100 nM) and LY294002 (25 $\mu \mathrm{M})$.

Quercetin treatment resulted in a dose-dependent increase in $\mathrm{LDH}$ release (Fig. 7B), an effect that was modulated by increasing insulin cotreatments. However, insulin additions were unable to prevent the cytotoxic response resulting from a quercetin concentration of $100 \mu \mathrm{M}$. This latter concentration of inhibitor also produced a pronounced elevation of CYP2E1 mRNA levels (Fig. 7C). The resulting CYP2E1 mRNA levels were equivalent to or above those measured in freshly isolated hepatocytes (as shown in 0-h slot-blot inset). In contrast, no increases in CYP2E1 were detected at quercetin concentrations of 10 and $25 \mu \mathrm{M}$, where near complete inhibition of $\mathrm{PKB}$ was recorded. Although quercetin treatments were without apparent impact on albumin mRNA expression (Fig. 7D), inhibitor concentrations of $100 \mu \mathrm{M}$ produced substantial cellular morphological perturbations (data not shown), similar to those observed with elevated doses of wortmannin and LY294002, and consistent with the elevated levels of LDH leakage detected (Fig. 7B).

Effect of Staurosporine on Reversal of Insulin-Mediated Suppression of CYP2E1 mRNA Expression. We tested further the hypothesis that increased CYP2E1 mRNA expression was associated with cytotoxicity due to treatment with exaggerated concentrations of PI3K inhibitors. Hepatocytes were treated with increasing concentrations of staurosporine, a relatively broad-spectrum protein kinase inhibitor, that also produces cytotoxic effects in various cell types (Janss et al., 1998). Cell treatments with staurosporine were conducted in a similar manner as already described for other inhibitors. Staurosporine additions actually stimulated PKB phosphorylation in a dose-dependent manner (Fig. 8A). This effect was only observed after a 30 -min pretreatment interval

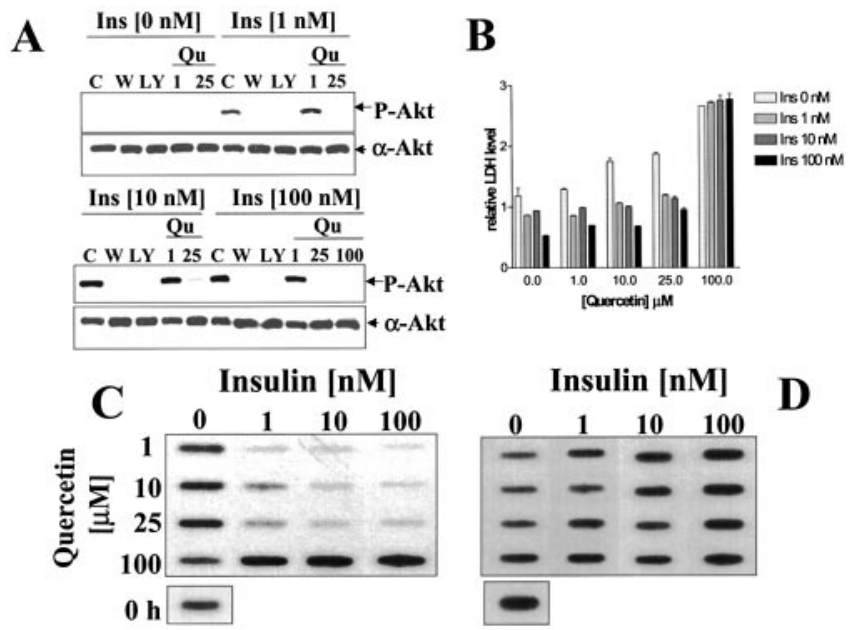

Fig. 7. Effect of quercetin concentration on insulin-mediated phosphorylation of PKB, LDH release, and suppression of CYP2E1 mRNA expression in primary rat hepatocytes. For explanation of treatments refer to Figs. 2 and 6. A, modulation of the level of insulin-stimulated phosphorylation of PKB (P-Akt) by wortmannin (100 nM), LY294002 $(25 \mu \mathrm{M})$, and quercetin $(1,25,100 \mu \mathrm{M})$. The level of immunoreactive protein was assessed with a phosphorylation-independent antibody to PKB ( $\alpha$-Akt). Level of LDH release (B), CYP2E1 (C), and albumin mRNA expression (D) are also shown. The corresponding levels of CYP2E1 and albumin mRNA expression in freshly isolated hepatocytes are shown for comparison in the two lower panels as $0 \mathrm{~h}$. followed by 30-min incubation with inhibitor in the presence or absence of insulin ( 0 or $10 \mathrm{nM}$ ). Longer treatments did not further enhance or inhibit PKB phosphorylation (data not shown). Staurosporine exhibits an $\mathrm{IC}_{50}$ value of $9 \mu \mathrm{M}$ with respect to PI3K inhibition (Meggio et al., 1995; Walker et al., 2000). Substantial stimulation of SAPK/JNK phosphorylation also resulted from the same concentrations of staurosporine that stimulated PKB phosphorylation (Fig. 8A). However, despite the inability of this agent to inhibit PKB, staurosporine treatments produced a marked accumulation of CYP2E1 mRNA. Even in the absence of insulin, staurosporine stimulated CYP2E1 mRNA levels, an effect that was saturated even at the lowest concentration of inhibitor used (10 nM, Fig. 8B). Treatment with 1000 nM the latter agent increased CYP2E1 mRNA levels above those measured in freshly isolated hepatocytes (see 0-h slot-blot inset, Fig. 8B). Despite the marked stimulatory effects on PKB, SAPK/JNK, and CYP2E1, staurosporine treatments resulted in no detectable disruption of albumin mRNA levels in the hepatocytes (Fig. 8C).

Morphological evidence of the cytotoxic effects of staurosporine on primary hepatocytes is presented in Fig. 9. Cellular deterioration is clearly evident at the 250 and $1000 \mathrm{nM}$ concentrations, where cytotoxicity also was observed (assessed by $\mathrm{LDH}$ release; data not shown). The disruption of cell shape and resulting perturbation of morphological integrity seen at elevated concentrations of staurosporine was even more dramatic than that observed with wortmannin, but similar to those noted for $100 \mu \mathrm{M}$ quercetin treatments (data not shown). The respective challenges were manifested in cells appearing ghost-like, with resultant loss of cytoplas-

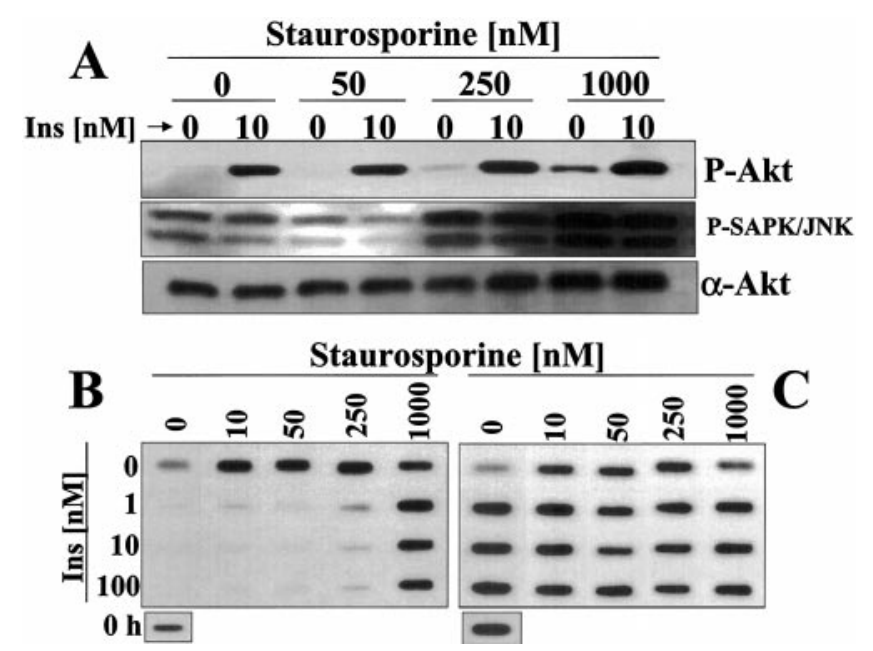

Fig. 8. Effect of staurosporine concentration on insulin-mediated phosphorylation of PKB and suppression of CYP2E1 mRNA expression. For general explanation of hepatocyte culture and treatments refer to Fig. 2. Otherwise, hepatocytes were treated with staurosporine (nM) for $1 \mathrm{~h}$ before treatment with insulin (nM). The phosphorylation status (A) of $\mathrm{PKB}$ and SAPK/JNK was assessed using phosphospecific antibodies directed against PKB (P-Akt, Ser473) and SAPK/JNK (Thr183/Tyr185) as stated under Experimental Procedures. The level of immunoreactive protein was assessed with a phosphorylation-independent antibody to PKB $(\alpha$-Akt). Treatments to assess mRNA levels were continued for a further $24 \mathrm{~h}$ and then total RNA was isolated and evaluated by slot-blot analysis as described under Experimental Procedures. CYP2E1 (B) and albumin mRNA (C) expression is shown. Ribosomal 18S RNA hybridization levels were used as normalization standards to demonstrate equal loading of RNA (data not shown). The corresponding levels of CYP2E1 and albumin mRNA expression in freshly isolated hepatocytes are shown for comparison in the two lower panels as $0 \mathrm{~h}$. 
mic detail. The compromised cellular morphology occurred despite the presence of insulin, an otherwise cytoprotective hormone in these cultures.

Evidence That Wortmannin Stimulates AP-1-Associated Stress Pathways. To further probe potential cellular stress effects associated with high concentrations of PI3K inhibitors, EMSA studies were conducted using a doublestranded oligonucleotide encompassing a consensus binding site for AP-1. As shown in Fig. 10, nuclear translocation of the AP-1 complex was observed $4 \mathrm{~h}$ after treatment with wortmannin $(10 \mu \mathrm{M})$ in the presence of $10 \mathrm{nM}$ insulin. Competition studies with excess unlabeled oligonucleotide and with a mutated oligonucleotide, further demonstrated the specificity of the interaction. Super shift analysis with an anti-c-Fos antibody authenticated the identity of the AP-1 complex. These results further demonstrate that general stress pathways are probably activated upon exposures of the hepatocytes to high levels of PI3K inhibitors.

\section{Discussion}

The experimental use of PI3K inhibitors, in particular wortmannin and LY294002, has enabled investigators to link various cellular processes with the involvement of PI3Kdependent pathways. These responses include mediation of the insulin-dependent induction and repression of hepatic gene expression involved in glycolysis and gluconeogenesis (Lemaigre and Rousseau, 1994), membrane autophagy, and apical plasma membrane internalization and turnover (Tuma et al., 1999). Although it is widely accepted that wortmannin is an irreversible and highly specific inhibitor of PI3K, with an $\mathrm{IC}_{50}$ value of $<50 \mathrm{nM}$ (Powis et al., 1994), this agent also has been reported to inhibit PI4K at high nM concentrations (Nakanishi et al., 1995) and, at micromolar concentrations, inhibits myosin light chain kinase (Powis et al., 1994). It is noteworthy that neither wortmannin nor LY294002 distinguish between the various isoforms of PI3K (Corvera and Czech, 1998), which may have differential in-
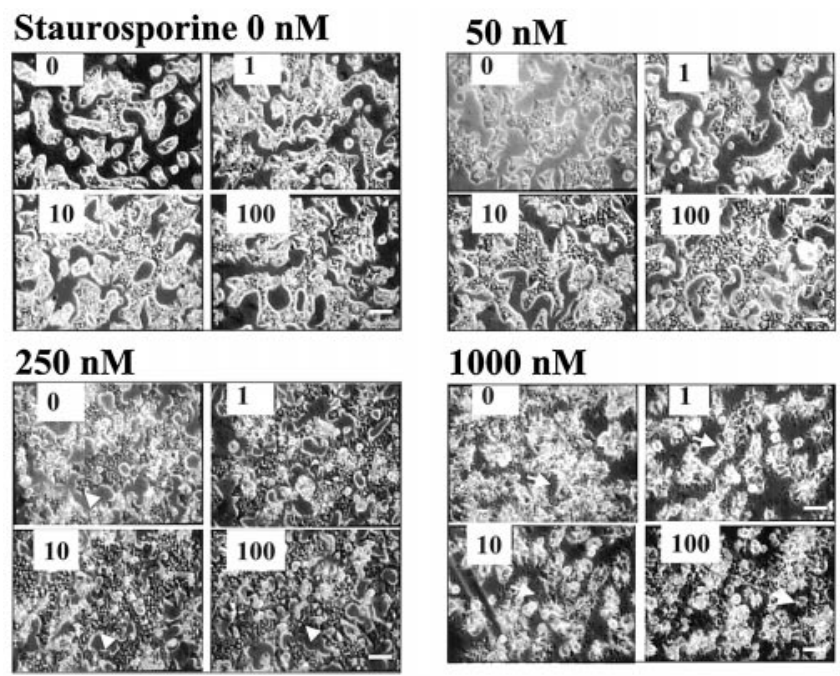

Fig. 9. Effect of staurosporine treatments on hepatocyte morphology. Primary rat hepatocytes were cultured and treated as stated in Fig. 1. The effect of staurosporine treatments $(0,50,250,1000 \mathrm{nM})$ for $24 \mathrm{~h}$, in the presence of insulin (depicted in top left corner of each panel as $0,1,10$, $100 \mathrm{nM}$ ), on the representative morphology (magnification, 20×) of primary rat hepatocytes is shown. Scale bars, $50 \mu \mathrm{m}$. Arrows indicate evidence of perturbed morphology attributed to cytotoxicity. hibition constants. LY294002 was originally synthesized (Vlahos et al., 1994) as a derivative of the naturally occurring bioflavonoid quercetin (Matter et al., 1992). Although quercetin possesses PI3K inhibitory potential $\left(\mathrm{IC}_{50}=3.8 \mu \mathrm{M}\right)$, it can also inhibit PI4K as well as other protein kinases $(\mathrm{Na}-$ kanishi et al., 1995). LY294002 $\left(\mathrm{IC}_{50}=1.4 \mu \mathrm{M}\right)$ is considered a more specific inhibitor of PI3K, but unlike wortmannin, it binds to PI3K in a competitive manner because it possesses a structure similar to that of ATP (Vlahos et al., 1994; Walker et al., 2000).

In this investigation, we used a well defined rat primary hepatocyte culture system that exhibits a highly differentiated phenotype and enables gene responsiveness that parallels the adult liver (Sidhu and Omiecinski, 1995a, 1998). We examined integrative effects of varying insulin concentrations and ECM environments on PI3K signaling pathways, hepatocyte stress responses and the modulation of CYP2E1 mRNA expression. A primary aim of this study was to examine the mechanism of insulin signaling with respect to its suppression of CYP2E1 mRNA expression in primary rat hepatocytes (Woodcroft and Novak, 1999a). In agreement with previous studies we found that physiological levels of insulin (1 $\mathrm{nM})$ did result in the suppression of CYP2E1 mRNA expression (Woodcroft and Novak, 1999a). However, in contrast to a previous report (Woodcroft and Novak, 1999b), our data demonstrate that these effects are independent of PI3K signaling. Rather, elevated concentrations of PI3K inhibitors produce a generalized cytotoxic response in hepatocytes that in turn stimulates CYP2E1 expression.

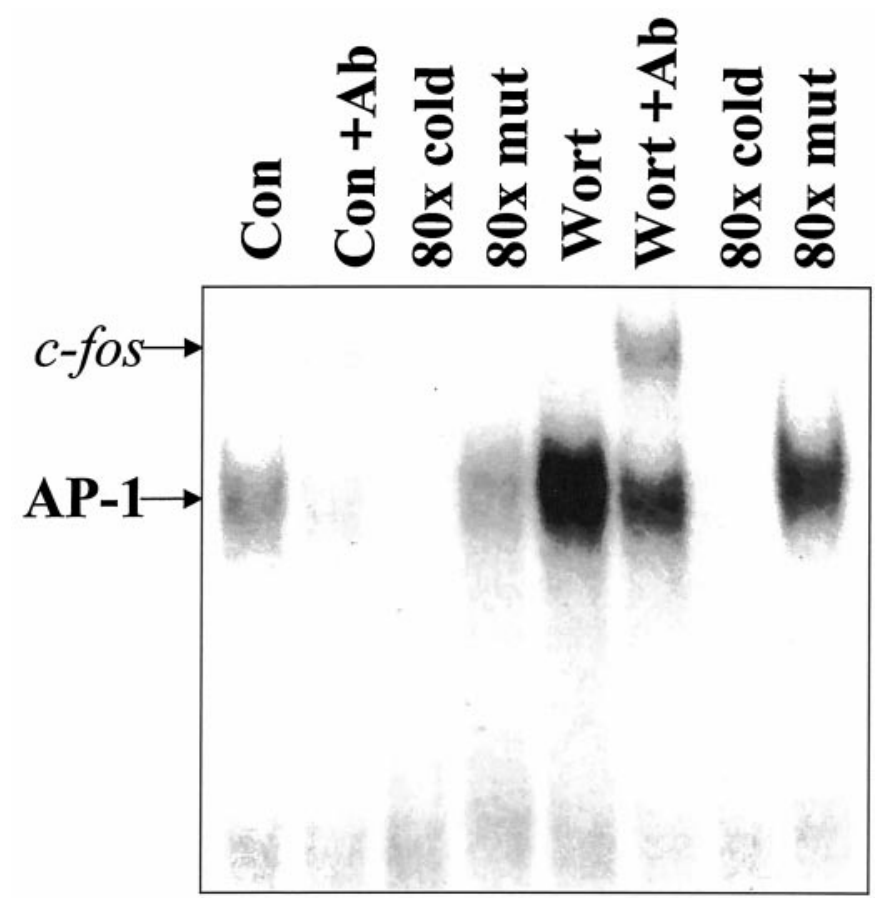

Fig. 10. EMSA analysis of wortmannin stimulation of AP-1 complex formation. Ten micrograms of nuclear protein extracted from untreated (Con) or wortmannin-treated (Wort) hepatocytes was incubated with an AP-1, double-stranded oligonucleotide as described under Experimental Procedures. Binding reactions done in the presence of polyclonal antibodies to $c$-fos (Con $+\mathrm{Ab}$ or Wort $+\mathrm{Ab}$ ) led to super shifted complexes. Binding reactions carried out with the addition of excess (80-fold) unlabeled probe $(80 \times$ cold $)$ resulted in complete elimination of binding complexes in both control and treated conditions. Incubation in excess unlabeled mutant probe $(80 \times$ mut) did not inhibit complex formation for either condition. 
In our experiments, wortmannin, at $50 \mathrm{nM}$, produced $>50 \%$ inhibition of $\mathrm{PKB}$ phosphorylation associated with 10 $\mathrm{nM}$ insulin stimulation. However, at this concentration the inhibitor was completely ineffective at reversing the suppressive effect of insulin on CYP2E1 mRNA expression. In fact, even a near saturating inhibition of $\mathrm{PKB}$ phosphorylation, achieved at $100 \mathrm{nM}$ wortmannin, did not effect the insulinassociated suppression of CYP2E1 mRNA levels. The latter response was only altered appreciably at wortmannin concentrations ranging from 1 to $10 \mu \mathrm{M}$. Similarly, dose-dependent treatment of cells with two other PI3K inhibitors tested, quercetin and LY294002, inhibited insulin-mediated stimulation of $\mathrm{PKB}$ phosphorylation in the low micromolar range. However, no detectable reversal of the insulin-mediated suppression of CYP2E1 mRNA expression was achieved using concentrations of these inhibitors that correlated with their PI3K effects. CYP2E1 reversal was only achieved at acutely cytotoxic concentrations of inhibitor $(\sim 100 \mu \mathrm{M})$, far in excess of that required to inhibit PKB phosphorylation. Furthermore, treatment of cells with staurosporine, a relatively nonspecific protein kinase inhibitor, resulted in a concentrationand time-dependent increase in cytotoxicity. The cytotoxic response from this agent was associated with pronounced elevation of CYP2E1 mRNA expression, despite occurring in the complete absence of inhibition of PKB phosphorylation.

The effects noted in this investigation provide compelling evidence to suggest that the PI3K inhibitors examined produce extended responses beyond that of inhibiting PI3Kdependent pathways. These results confirm our general conclusion that the effects of these inhibitors on CYP2E1 expression are independent of a PI3K pathway and are likely to be associated with cytotoxicity. This hypothesis was substantiated by the marked morphological perturbations observed in the hepatocytes at elevated levels of inhibitor, as well as by increased $\mathrm{LDH}$ release from the cells.

At micromolar concentrations, wortmannin is known to inhibit DNA protein kinases (Boulton et al., 1999) and exerts cytotoxic effects in a number of cell types. In this capacity, the inhibitor has been used at high micromolar concentrations to sensitize cells to the cytotoxic effects of ionizing radiation (Price and Youmell, 1996), receptor-directed-toxin chimeras (Davol et al., 1999), and chemical treatment (Christodoulopoulos et al., 1998). Similar sensitization effects have also been reported for quercetin (Agullo et al., 1994) and stauorsporine (Janss et al., 1998).

Several recent studies have demonstrated that various cellular stresses, including UV irradiation, osmotic shock, and chemical stress (Woodgett et al., 1996) result in the striking and rapid phosphorylation of the SAPK/JNK and p38 MAPK cascades. This has been correlated with the subsequent activation of various transcription factors (Janknecht and Hunter, 1997), which in turn have been shown to activate genes involved in a number of cellular processes, including apoptosis (Woodgett et al., 1996).

We examined whether the cytotoxicity associated with inhibitors examined, and the resulting elevation of CYP2E1 expression, was due to the activation of SAPK/JNK and/or p38 MAPK phosphorylation. Our findings demonstrate that both wortmannin and LY294002 stimulated SAPK/JNK and p38 MAPK phosphorylation in a concentration-dependent manner that paralleled the inhibition of PKB phosphorylation. These represent novel findings, not previously reported.
Staurosporine also stimulated SAPK/JNK and p38 MAPK phosphorylation (data not shown) in treated hepatocytes, at similar concentrations that activated $\mathrm{PKB}$ phosphorylation (250-1000 nM) and stimulated CYP2E1 mRNA levels. Although treatment with the protein synthesis inhibitor anisomycin (Sidhu and Omiecinski, 1998) resulted in a potent activation of SAPK/JNK, it was completely ineffective in producing either cytotoxicity or modulation of CYP2E1 expression levels. These latter observations may not necessarily exclude an involvement of the SAPK/JNK or p38 MAPK pathways as modulators of certain kinds of chemical stress, cytotoxicity, and/or CYP2E1 expression in hepatocytes. They do suggest that activation of the SAPK/JNK and/or p38 MAPK pathways represents a consequence, rather than a direct cause, of PI3K inhibitor-induced cytotoxicity in primary rat hepatocytes. Our findings that wortmannin treatments stimulated SAPK/JNK phosphorylation, as well as nuclear recruitment of the AP-1 complex, are also intriguing. Further studies are required to determine the biological consequences associated with these effects.

Elevation of CYP2E1 expression, similar to that achieved with high levels of PI3K inhibitors, also has been observed with other chemically diverse agents, such as ethanol and pyridines, agents that similarly induce or enhance cytotoxicity (Anundi and Lindros, 1992; Niemela et al., 1998) in liver. The insulin-mediated antagonism of CYP2E1 induction observed with such agents may therefore occur through antiapoptotic or cytoprotective properties associated with the hormone. Further analysis of the potential cross talk between insulin and SAPK/JNK and/or p38 MAPK pathways should shed more light on the specific mechanisms of cytotoxicity involved in these responses and lead to a more precise understanding of the toxicant-associated regulation of CYP2E1 expression in both acute and chronic chemical challenge in adult liver.

\section{Acknowledgments}

We gratefully acknowledge the critical reading and suggestions of Richard Ramsden and Scott Auerbach.

\section{References}

Agullo G, Gamet L, Besson C, Demigne C and Remesy C (1994) Quercetin exerts a preferential cytotoxic effect on active dividing colon carcinoma HT29 and Caco-2 cells. Cancer Lett 87:55-63.

Alessi DR, Andjelkovic M, Caudwell B, Cron P, Morrice N, Cohen P and Hemmings BA (1996) Mechanism of activation of protein kinase B by insulin and IGF-1. EMBO J 15:6541-6551.

Anundi I and Lindros KO (1992) Evidence for cytochrome P450 2E1-mediated toxicity of $N$-nitrosodimethylamine in cultured perivenous hepatocytes from ethanol treated rats. Pharmacol Toxicol 70:453-458.

Avruch J (1998) Insulin signal transduction through protein kinase cascades. Mol Cell Biochem 182:31-48.

Bellacosa A, Testa JR, Staal SP and Tsichlis PN (1991) A retroviral oncogene, akt, encoding a serine-threonine kinase containing an SH2-like region. Science (Wash DC) 254:274-277.

Bissell DM, Caron JM, Babiss LE and Friedman JM (1990) Transcriptional regulation of the albumin gene in cultured rat hepatocytes. Role of basement-membrane matrix. Mol Biol Med 7:187-197.

Boulton S, Kyle S and Durkacz BW (1999) Interactive effects of inhibitors of poly(ADP-ribose) polymerase and DNA-dependent protein kinase on cellular responses to DNA damage. Carcinogenesis 20:199-203.

Christodoulopoulos G, Muller C, Salles B, Kazmi R and Panasci L (1998) Potentiation of chlorambucil cytotoxicity in B-cell chronic lymphocytic leukemia by inhibition of DNA-dependent protein kinase activity using wortmannin. Cancer Res 58:1789-1792.

Chuang LM, Hausdorff SF, Myers MG Jr, White MF, Birnbaum MJ and Kahn CR (1994) Interactive roles of Ras, insulin receptor substrate-1, and proteins with Src homology-2 domains in insulin signaling in Xenopus oocytes. J Biol Chem 269: $27645-27649$.

Corvera S and Czech MP (1998) Direct targets of phosphoinositide 3-kinase products in membrane traffic and signal transduction. Trends Cell Biol 8:442-446. 
Davol PA, Bizuneh A and Frackelton AR Jr (1999) Wortmannin, a phosphoinositide 3-kinase inhibitor, selectively enhances cytotoxicity of receptor-directed-toxin chimeras in vitro and in vivo. Anticancer Res 19:1705-1713.

Dong ZG, Hong JY, Ma QA, Li DC, Bullock J, Gonzalez FJ, Park SS, Gelboin HV and Yang CS (1988) Mechanism of induction of cytochrome P-450ac (P-450j) in chemically induced and spontaneously diabetic rats. Arch Biochem Biophys 263:29-35.

Franke TF, Kaplan DR and Cantley LC (1997) PI3K: downstream AKTion blocks apoptosis. Cell 88:435-437.

Hara K, Yonezawa K, Sakaue H, Ando A, Kotani K, Kitamura T, Kitamura Y, Ueda $\mathrm{H}$, Stephens L and Jackson TR (1994) 1-Phosphatidylinositol 3-kinase activity is required for insulin-stimulated glucose transport but not for RAS activation in CHO cells. Proc Natl Acad Sci USA 91:7415-7419.

Janknecht R and Hunter T (1997) Activation of the Sap-1a transcription factor by the c-Jun N-terminal kinase (JNK) mitogen-activated protein kinase. J Biol Chem 272:4219-4224

Janss AJ, Levow C, Bernhard EJ, Muschel RJ, McKenna WG, Sutton L and Phillips PC (1998) Caffeine and staurosporine enhance the cytotoxicity of cisplatin and camptothecin in human brain tumor cell lines. Exp Cell Res 243:29-38.

Kandel ES and Hay N (1999) The regulation and activities of the multifunctiona serine/threonine kinase Akt/PKB. Exp Cell Res 253:210-229.

Kimball SR, Horetsky RL and Jefferson LS (1995) Hormonal regulation of albumin gene expression in primary cultures of rat hepatocytes. Am J Physiol 268:E6-E14.

Klippel A, Kavanaugh WM, Pot D and Williams LT (1997) A specific product of phosphatidylinositol 3-kinase directly activates the protein kinase Akt through its pleckstrin homology domain. Mol Cell Biol 17:338-344.

Kraner JC, Lasker JM, Corcoran GB, Ray SD and Raucy JL (1993) Induction of $\mathrm{P} 4502 \mathrm{E} 1$ by acetone in isolated rabbit hepatocytes. Role of increased protein and mRNA synthesis. Biochem Pharmacol 45:1483-1492.

Lemaigre FP and Rousseau GG (1994) Transcriptional control of genes that regulate glycolysis and gluconeogenesis in adult liver. Biochem $J$ 303:1-14.

Lieber CS (1997) Cytochrome P-4502E1: its physiological and pathological role. Physiol Rev 77:517-544

Matter WF, Brown RF and Vlahos CJ (1992) The inhibition of phosphatidylinositol 3-kinase by quercetin and analogs. Biochem Biophys Res Commun 186:624-631.

Meggio F, Donella D A, Ruzzene M, Brunati A M, Cesaro L, Guerra B, Meyer T, Mett H, Fabbro D and Furet P (1995) Different susceptibility of protein kinases to staurosporine inhibition. Kinetic studies and molecular bases for the resistance of protein kinase CK2. Eur J Biochem 234:317-322.

Nakanishi S, Catt KJ and Balla T (1995) A wortmannin-sensitive phosphatidylinositol 4-kinase that regulates hormone-sensitive pools of inositolphospholipids. Proc Natl Acad Sci USA 92:5317-5321.

Nelson DR, Koymans L, Kamataki T, Stegeman JJ, Feyereisen R, Waxman DJ, Waterman MR, Gotoh O, Coon MJ, Estabrook RW, et al (1996) P450 superfamily: update on new sequences, gene mapping, accession numbers and nomenclature. Pharmacogenetics 6:1-42.

Niemela O, Parkkila S, Pasanen M, Iimur Y, Bradford B and Thurman RG (1998 Early alcoholic liver injury: formation of protein adducts with acetaldehyde and lipid peroxidation products, and expression of CYP2E1 and CYP3A. Alcohol Clin Exp Res 22:2118-2124.

O'Brien RM and Granner DK (1996) Regulation of gene expression by insulin. Physiol Rev 76:1109-1161.
Pessin JE and Saltiel AR (2000) Signaling pathways in insulin action: molecular targets of insulin resistance. J Clin Invest 106:165-169.

Powis G, Bonjouklian R, Bergoren MM, Gallegos A, Abraham R, Ashendel C, Zalkow L, Matter WF, Dodge J and Grindey G (1994) Wortmannin, a potent and selective inhibitor of phosphatidylinositol-3-kinase. Cancer Res 54:2419-2423.

Price BD and Youmell MB (1996) The phosphatidylinositol 3-kinase inhibitor wortmannin sensitizes murine fibroblasts and human tumor cells to radiation and blocks induction of p53 following DNA damage. Cancer Res 56:246-250.

Sidhu JS, Farin FM and Omiecinski CJ (1993) Influence of extracellular matrix overlay on phenobarbital-mediated induction of CYP2B1, 2B2, and 3A1 genes in primary adult rat hepatocyte culture. Arch Biochem Biophys 301:103-113.

Sidhu JS and Omiecinski CJ (1995a) cAMP-associated inhibition of phenobarbitalinducible cytochrome $\mathrm{P} 450$ gene expression in primary rat hepatocyte cultures. $J$ Biol Chem 270:12762-12773.

Sidhu JS and Omiecinski CJ (1995b) Modulation of xenobiotic-inducible cytochrome P450 gene expression by dexamethasone in primary rat hepatocytes. Pharmacogenetics 5:24-36.

Sidhu JS and Omiecinski CJ (1998) Protein synthesis inhibitors exhibit a nonspecific effect on phenobarbital-inducible cytochrome $\mathrm{P} 450$ gene expression in primary rat hepatocytes. J Biol Chem 273:4769-4775.

Sidhu JS and Omiecinski CJ (1999) Insulin-mediated modulation of cytochrome $\mathrm{P} 450$ gene induction profiles in primary rat hepatocyte cultures. $J$ Biochem Mol Toxicol 13:1-9.

Tuma PL, Finnegan CM, Yi JH and Hubbard AL (1999) Evidence for apical endocytosis in polarized hepatic cells: phosphoinositide 3-kinase inhibitors lead to the lysosomal accumulation of resident apical plasma membrane proteins. J Cell Biol 145:1089-1102.

Vlahos CJ, Matter WF, Hui KY and Brown RF (1994) A specific inhibitor of phosphatidylinositol 3-kinase, 2-(4- morpholinyl)-8-phenyl-4H-1-benzopyran-4-one (LY294002). J Biol Chem 269:5241-5248.

Walker EH, Pacold ME, Perisic O, Stephens L, Hawkins PT, Wymann MP and Williams RL (2000) Structural determinants of phosphoinositide 3-kinase inhibition by wortmannin, LY294002, quercetin, myricetin, and staurosporine. Mol Cell 6:909-919.

Woodcroft KJ and Novak RF (1999a) Insulin differentially affects xenobioticenhanced, cytochrome P-450 (CYP)2E1, CYP2B, CYP3A, and CYP4A expression in primary cultured rat hepatocytes. J Pharmacol Exp Ther 289:1121-1127.

Woodcroft KJ and Novak RF (1999b) The role of phosphatidylinositol 3-kinase, Src kinase, and protein kinase A signaling pathways in insulin and glucagon regulation of CYP2E1 expression. Biochem Biophys Res Commun 266:304-307.

Woodgett JR, Avruch J and Kyriakis J (1996) The stress activated protein kinase pathway. Cancer Surv 27:127-138.

Zaluzny L, Farrell GC and Murray M (1990) Effect of genetic obesity and experimental diabetes on hepatic microsomal mixed function oxidase activities. $J$ Gas troenterol Hepatol 5:256-263.

Send reprint requests to: Curtis J. Omiecinski, Ph.D, Department of Environmental Health, University of Washington, 4225 Roosevelt Way NE, \#100, Seattle, WA 98105-6099. E-mail: cjo@u.washington.edu 